Supporting Information

\title{
Extrusion-based 3D bioprinting of gradients of stiffness, cell density and immobilized peptide using thermogelling hydrogels
}

Merve Kuzucü, Grace Vera ${ }^{\#}$, Marco Beaumont ${ }^{\dagger}$, , Sascha Fischer ${ }^{\#}$, Pan Wei ${ }^{\#}$, V. Prasad Shastrii ${ }^{\#, \$}$ and Aurelien Forget ${ }^{\# *}$

\#Institute for Macromolecular Chemistry, University of Freiburg, Stefan-MeierStr. 31, 79104 Freiburg, Germany

$†$ School of Chemistry and Physics, Queensland University of Technology, 2

George St, Brisbane City, QLD 4000, Australia

\$Institute of Chemistry of Renewable Resources, University of Natural Resources and Life Sciences, (BOKU), Konrad-Lorenz-Straße 243430 Tulln, Austria

†IOSS, Centre for Cell Signalling Studies, Schänzlestr. 18, 79104 Freiburg, Germany

* Correspondence to: aurelien.forget@makro.uni-freiburg.de 


\section{$\underline{\text { Table of Contents }}$}

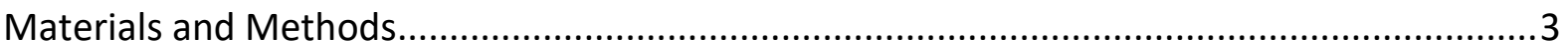

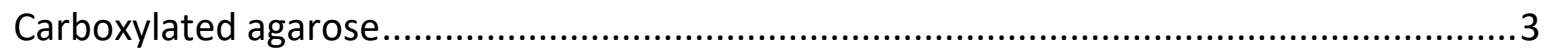

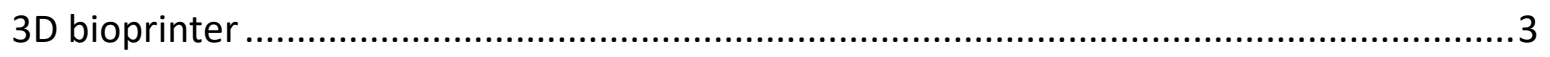

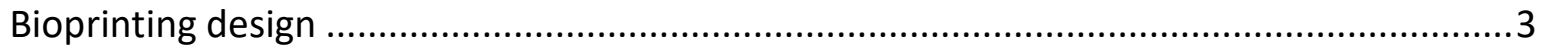

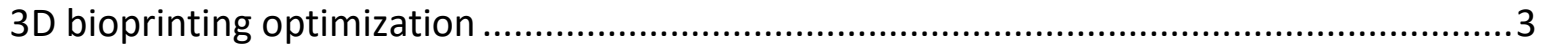

Maleimide functionalized agarose .......................................................................... 4

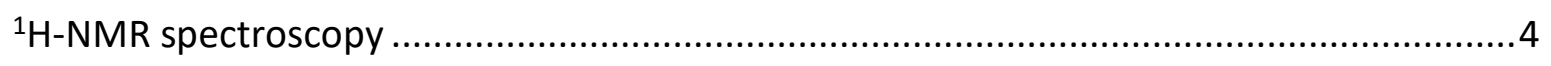

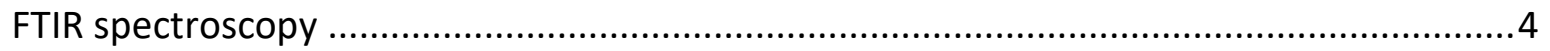

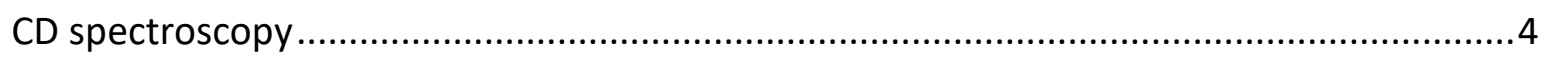

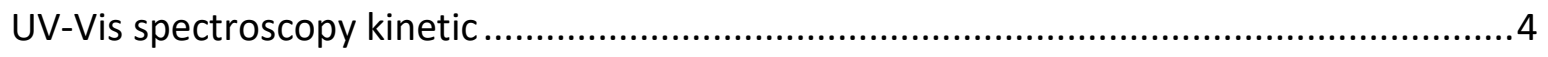

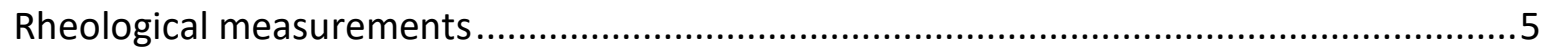

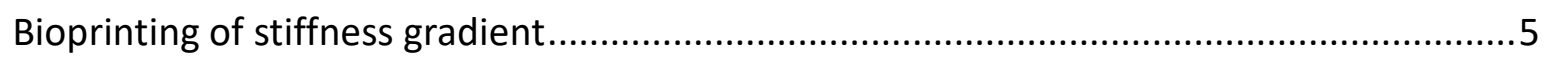

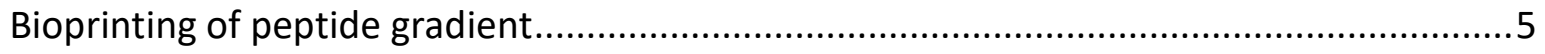

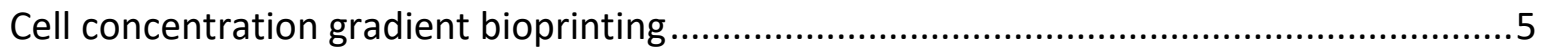

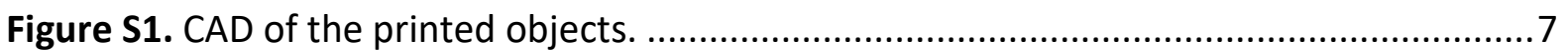

Figure S2. Optimization of printing parameter. ........................................................... 8

Figure S3. Technical drawing of the mixing head ..............................................................

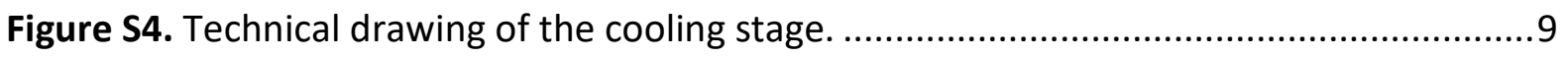

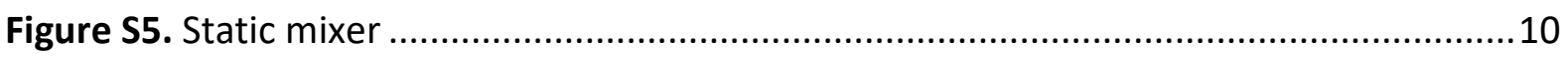

Table S1. Flow rate parameters for 2D Bioprinting of graded mechanical properties ...........11

Table S2. Flow rate parameters for 3D Bioprinting of graded mechanical properties ...........11

Table S3. Flow rate parameters for 2D Bioprinting of graded concentration of cells. ...........11

Table S4. Flow rate parameters for 3D Bioprinting of graded concentration of cells. ...........11

Figure S6. Physico-chemical analysis of the soft carboxylated agarose (CA)........................12

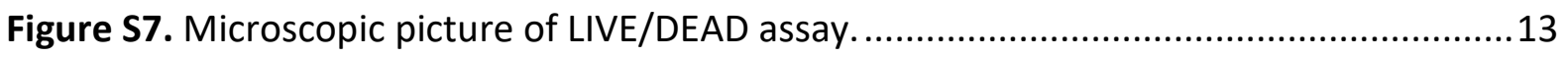




\section{Materials and Methods}

\section{Carboxylated agarose}

Carboxylated agarose was synthesized as previously reported. ${ }^{14}$ One gram of native agarose (NA) type

1 (GeneON, Germany) was transferred into a three-necked round bottom flask, equipped with a mechanical stirrer and $\mathrm{pH}$ meter. The reactor was heated to $90^{\circ}$ to dissolve the agarose in water (500 $\mathrm{mL}$ ) and then cooled to $0{ }^{\circ} \mathrm{C}$ under mechanically stirring to prevent the solution for gelling. The reactor was then charged with TEMPO $(0.160 \mathrm{mmol}, 20.6 \mathrm{mg}), \mathrm{NaBr}(0.9 \mathrm{mmol}, 0.1 \mathrm{~g})$, and $\mathrm{NaOCl}(2.5 \mathrm{~mL}$, $15 \% \mathrm{vol} / \mathrm{vol}$ solution) under vigorous stirring. The $\mathrm{pH}$ of the solution was adjusted to $\mathrm{pH} 10.8$ throughout the reaction, and the degree of carboxylation was controlled by the addition of predetermined volumes of $\mathrm{NaOH}$ solution $(0.5 \mathrm{M})$. At the end of the reaction, $\mathrm{NaBH}_{4}(0.1 \mathrm{~g})$ was added, and the solution was acidified to $\mathrm{pH} 8$ and stirred for $1 \mathrm{~h}$. The CA was precipitated by sequential addition of $\mathrm{NaCl}(12 \mathrm{~g})$ and ethanol $(500 \mathrm{~mL})$, and the solid was collected by vacuum filtration and extracted using ethanol. Residual ethanol was removed by extensive dialysis against water and the CA was obtained as a white solid upon freeze-drying overnight. In this study, CA of three different degrees of carboxylation were used to access hydrogels from 2 to $8 \% \mathrm{w} / \mathrm{v}$. Final optimized formulation of CA hydrogel were selected with $6 \% \mathrm{w} / \mathrm{v}$ with shear modulus at $1 \mathrm{~Hz}$ of $1520 \pm 106 \mathrm{~Pa}$ (soft), $2234 \pm 149 \mathrm{~Pa}$ (medium) and $3745 \pm 93 \mathrm{~Pa}$ (stiff). TEMPO, $\mathrm{NaBr}, \mathrm{NaOCl}, \mathrm{NaCl}$ and $\mathrm{NaBH}_{4}$ were obtained from Sigma Aldrich (Germany) and used as received.

\section{$3 D$ bioprinter}

The bioprinter was costumed build and based on a Magician Robotic arm (DOBOT, China). A custombuilt mixing head with two openings was machined from stainless steel (SI-Figure 1). Two openings (10-32 UNF) of $4.1 \mathrm{~mm}$ and $2 \mathrm{~mm}$ inner diameter channels at an angle of $135^{\circ}$ with respect to one another converged into a $2.5 \mathrm{~mm}$ diameter channel of $7 \mathrm{~mm}$ length. In this channel a 6-elements static mixer with $15 \mathrm{~mm}$ length and $1.5 \mathrm{~mm}$ diameter static mixer was positioned (Misch- und Dosiertechnik $\mathrm{GmbH}$, Germany) (SI-Figure 5). The mixing head was fitted with a barbed adapter on the feeding inputs and a Luer-lock at the output (Cole Parmer, USA). Two holes of $4 \mathrm{~mm}$ diameter each were drilled on the top of the head to accommodate a PT100 thermal sensor (Conrad, Germany) and a heating cartridge (Conrad, Germany). The heating system was controlled by a Thermo controller STC100. The tubes ( $4 \times 2$, polyurethane tubing, Cole Parmer, USA) carrying the bioink from the syringes to the mixing head were enfolded into a heating pad (New Era, USA). The temperature controller for the heating pads consists of a primary and secondary output. The primary exits are connected to cover the syringes, and the secondary outputs are connected to cover the pipes. The $10 \mathrm{~mL}$ syringes (Luer lock tip, Terumo, Japan) were kept at $40^{\circ} \mathrm{C}$ by two heating pads controlled by the New Era controller. The syringes were loaded with the printing media and positioned on the syringe pumps (Cole Parmer, USA). On the printing area below the arm of the robotic arm, a water-cooled stage was positioned as described earlier. ${ }^{15}$ The custom-built stage was built of poly(carbonate) (PC) receptacle with two openings equipped with a tube connector to let the water flow through. On the PC bottom was glued an aluminum plate that was machined with threads to hold petri dish of different sizes. Cold water was circulated at $4^{\circ} \mathrm{C}$ through the printing stage to allow rapid gelation of the CA bioink.

\section{Bioprinting design}

The printed design was drawn on Inventor (Autodesk, 2018) and exported as an STL file. The files were then converted into G-code using Repetier Host (Hot-World GmbH) and Slic3R (Open Source). Printing parameters of the robot such as layer thickness and speed were controlled on Repetier Host. Two designs were created: (1) 2D serpentine line and (2) a 3D cylinder (SI-Figure 2).

\section{$3 D$ bioprinting optimization}

Various concentration of CA soft, medium, and stiff were prepared in phosphate-buffered saline solution (Gibco) ranging from $2 \% \mathrm{w} / \mathrm{v}$ to $8 \% \mathrm{w} / \mathrm{v}$. Each formulation was printed, and the dimension of 
the printed object was measured and compared with the dimension of the designed object. For the planar object, a picture of the object was taken with a reference dimension that allowed us to convert pixel into metric units. Using Image ( $\mathrm{NIH}$, Bethesda, Maryland, USA) the diameter of the line was measured in pixel and then converted into a metric value. Each measurement is the average of nine printed samples and error bars represent the standard deviation. In case of the cylinders, the dimensions were measured with calipers. The outer diameter, inner diameter, and height of printed samples $(n=9)$ were measured, and the dimensions were compared with the input design to determine the deviation from the prescribed dimensions.

\section{Maleimide functionalized agarose}

Soft CA was dissolved in $0.1 \mathrm{M}$ 2-(N-morpholino)ethanesulfonic acid buffer solution (pH 4) (Sigma Aldrich, Germany) in a round bottom flask by heating in a water bath at $90^{\circ} \mathrm{C}$ for 5 minutes under mechanical stirrer in a laminar flow. The maximum concentration of CA in the MES solution was 0.5 $\mathrm{w} / \mathrm{v} \%$ to avoid gel formation. Once the CA was dissolved the solution was cooled down to room temperature, and a 10-fold molar excess of EDC (Sigma Aldrich, Germany) dissolved in MES solution was added to the main solution to activate the carboxylic groups in the CA. After 45 minutes of activation, maleimide ( $1 \mathrm{mmol} / \mathrm{g} \mathrm{CA}$ ) was dissolved in MES solution and added to the main solution, and the solution could react for 24 hours at room temperature under mechanical stirring. The functionalized CA was purified by dialysis using a cellulose acetate membrane ( $3.5 \mathrm{kDa}$, MWCO, Spectra Pore, USA) against distilled water for three days. The dialysis waster was changed twice a day at 6-hour intervals. Finally, the sample was freeze-dried using liquid nitrogen in a round bottom flask and dried for 24 hours under vacuum (0.1 mbar).

${ }^{1} H$-NMR spectroscopy

${ }^{1} \mathrm{H}$-NMR spectra were recorded at $25{ }^{\circ} \mathrm{C}$, with water suppression on an Advance spectrometer at 300 $\mathrm{MHz}$ (Bruker, Germany). Samples' solutions $\left(10 \mathrm{mg} / \mathrm{ml}\right.$ ) were prepared with deuterium oxide $\left(\mathrm{D}_{2} \mathrm{O}\right)$ by heating in a water bath at $90^{\circ} \mathrm{C}$ until a clear solution was obtained. All chemical shifts are reported in ppm relative to $\mathrm{D}_{2} \mathrm{O}(\delta=4.8 \mathrm{ppm})$.

\section{FTIR spectroscopy}

Fourier transformed infrared (FTIR) spectrometer Vector 22 (Bruker, Germany) was used to record the IR spectra. Samples were mixed with potassium bromide $(0.25 \mathrm{w} / \mathrm{w} \%)$ and then pressed for 2 minutes at 10 ton into discs. All samples were measured using 30 scans with a resolution of $4 \mathrm{~cm}^{-1}$ in the $4000-$ $600 \mathrm{~cm}^{-1}$ range.

\section{$C D$ spectroscopy}

Circular dichroism (CD) spectra of functionalized carboxylated agarose $(0.5 \mathrm{mg} / \mathrm{ml})$ in a wavelength range of 180 to $300 \mathrm{~nm}$ were recorded on a J-810 CD spectrometer (Jasco, Japan). Measurements were carried out in a $1 \mathrm{~cm}$ path length quartz cuvette at $25^{\circ} \mathrm{C}$ and the spectra is an average of four scans.

\section{UV-Vis spectroscopy kinetic}

UV-Vis measurements were collected using a Thermo Evolution 201 spectrometer (ThermoFischer, Germany). Quartz cuvettes were used for all samples, and the recorded absorbance was from 800$190 \mathrm{~nm}$ and the baseline was corrected at $750 \mathrm{~nm}$. The kinetics of the thiol-ene reaction was performed with a 5-fold molar excess of CA-Maleimide $(6 \mathrm{mg} / \mathrm{ml})$. CA-Maleimide was dissolved in distilled water and the cysteine peptide (CRGDS, $593.61 \mathrm{~g} / \mathrm{mol}, 98.82 \%$, Proteogenix, France) was dissolved in PBS $(0.2 \mathrm{mg} / \mathrm{ml})$. Measurements were recorded at $37{ }^{\circ} \mathrm{C}$ with a magnetic stirrer for one hour. 


\section{Rheological measurements}

Rheology experiments were performed with a Kinexus Pro rheometer (Malvern Panalytical, UK). Samples were dissolved in distilled water $(2 \mathrm{w} / \mathrm{v} \%)$ in a water bath at $90^{\circ} \mathrm{C}$ for 5 minutes until a clear solution was obtained. All samples were stored at $4{ }^{\circ} \mathrm{C}$ for $24 \mathrm{~h}$ to allow gel formation before the measurements. A cone disc of $40 \mathrm{~mm}$ and $1^{\circ}$ was used. The test was performed at $1 \mathrm{~Hz}$ shear frequency and room temperature. Each point is a mean of the measurement of three different experiments.

\section{Bioprinting of stiffness gradient}

The objects with graded mechanical properties were prepared by dissolving each of the soft, medium, and stiff $C A$ in $10 \mathrm{ml}$ syringes at $6 \% \mathrm{w} / \mathrm{v}$. The syringes and tubes are wrapped in heating pads and the cold water is circulating through the cold stage. A static mixer (SI-Figure 5) was added in the mixing head before the extrusion nozzle. The static mixer was a polypropylene 6-elements Helix Mixer KSystem 0001-9998-0208MKHX (Misch- und Dosiertechnik GmbH, Germany) with a length of $15 \mathrm{~mm}$ and diameter of $1.5 \mathrm{~mm}$. The extrusion flow rate of each syringe is varied during the printing and set according to SI-Table 1 for the 2D printing and SI-Table 2 for the 3D printing. The mechanical properties of the printed object were determined using a Texture Analyzer TA-XT2i (Stable MicroSystems Ltd., Surrey, UK). The analyzer was equipped with a self-made cylinder probe ( $2 \mathrm{~mm}$ thickness, $3.14 \mathrm{~mm}^{2}$ area). The measurement was started at a pre-force of $0.005 \mathrm{~N}$ and conducted at a speed of $0.5 \mathrm{~mm} / \mathrm{s}$ till a maximum compressive strain of $20 \%$. The indentation elastic modulus was calculated in the linear regime (ca. 2-6\% strain). The 3D hydrogel samples were sliced parallel to z-axis (from bottom to top) and measurements were conducted in the z-axis direction, average values of hardness and modulus were calculated from three independent slices of a single hydrogel sample

\section{Bioprinting of peptide gradient}

The objects with graded mechanical properties were prepared by dissolving the soft CA in $10 \mathrm{ml}$ syringes at $6 \% \mathrm{w} / \mathrm{v}$ and the CRGDS-FITC peptide at $1 \mathrm{mg} / \mathrm{mL}$ in PBS (Gibco, Germany). The syringes and tubes are wrapped in heating pads and the cold water is circulating through the cold stage. The extrusion flow rate of each syringe is varied during the printing and set according to SI-Table 3 for the 2D printing and SI-Table 4 for the 3D printing. A static mixer (SI-Figure 5) was added in the mixing head before the extrusion nozzle. The static mixer was a polypropylene 6-elements Helix Mixer KSystem 0001-9998-0208MKHX (Misch- und Dosiertechnik GmbH, Germany) with a length of $15 \mathrm{~mm}$ and diameter of $1.5 \mathrm{~mm}$. Peptide attachment was tested by printing with peptide at the same concentration a soft hydrogel and a soft hydrogel functionalized with maleimide. Each of the object (Soft CA and Soft CA-maleimide) printed with peptide was submerged in deionized water for $24 \mathrm{~h}$ and the supernatant was collected at a different time interval and UV-vis spectrometry was performed to measure the amount of peptide diffused outside of the printed object. The gradient of peptide immobilized on the hydrogel was measured using Image Image analysis software. Photography of the sample was acquired, then converted in a black and white picture and the pixel intensity was calculated.

\section{Cell concentration gradient bioprinting}

As a proof of concept toward the fabrication of objects with cell gradients, HEK293 cells were printed. HEK293 were cultured in DMEM supplemented with $10 \%$ (vol/vol) FBS and 1\% Penicillin-StreptomycinAmphotericin $\mathrm{B}$ mixture in a humidified incubator at $5 \% \mathrm{CO}_{2}$ and $37^{\circ} \mathrm{C}$. Cell viability was assessed by adding Hoechst $33342(1 \mu \mathrm{L} / \mathrm{mL})$. Cells were trypsinized and loaded in $10 \mathrm{ml}$ syringes at a concentration of $4 \times 10^{6}$ cells $/ \mathrm{mL}$. The cells solution was mixed in the printer with the CA soft formulation according to the flow rate sequences described in SI-Table 3 and SI-Table 4. A static mixer (SI-Figure 5) was added in the mixing head before the extrusion nozzle. The static mixer was a polypropylene 6-elements Helix Mixer K-System 0001-9998-0208MKHX (Misch- und Dosiertechnik GmbH, Germany) with a length of $15 \mathrm{~mm}$ and diameter of $1.5 \mathrm{~mm}$. The incorporation of the cells in the 2D printed object was directly 
assessed on a microscope Axio Vision (Zeiss, Germany) with a 10x objective. Three samples of the printed 3D objects were placed on a microscopy slide and pictures of the side of the cylinder were imaged and stitched together to form a complete image. The number of cells per field of view was counted using Image J. At each position, pictures of at different focal planes were acquired, the file was then turned into 8-bit grayscale, threshold and then the particles analyzer tool of ImageJ was used to count the number of cells.

\section{Cell viability}

HEK293 cells suspended at the concentration of $4 \times 1$ cells / $\mathrm{mL}$ in DMEM with $10 \%$ vol/vol FBS and $1 \%$ Penicillin-Streptomycin Amphotericin B mixture were mixed with the CA soft at different flow rate from 0.1 to $0.12 \mathrm{~mL} / \mathrm{min}$. In total $100 \mu \mathrm{L}$ was extruded in a 96-wellplate (Nunc, Germany). The cell viability was then directly assay. We used a Live/Dead Assay kit (Life Technologies, Germany) immediately after printing. The staining solution was created by adding $0.2 \mu \mathrm{L}$ calcein AM (Component A) and $1 \mu \mathrm{L}$ ethidium homodimer-1 (Component B) into $1 \mathrm{~mL}$ DPBS. Each sample was stained with $100 \mu \mathrm{L}$ of the staining solution then incubated 30 minutes at $20-25^{\circ} \mathrm{C}$. Optical microscopy images were obtained on a Zeiss Observer A1 (Carl Zeiss, Germany). The results were analyzed by Image J. The number of cells per field of view was calculated. The calculated percentage of living cells was normalized to a sample that was not bioprinted. Statistical analysis was done using Graphpad unpair t-test calculator (GraphPad Software, USA). 
A

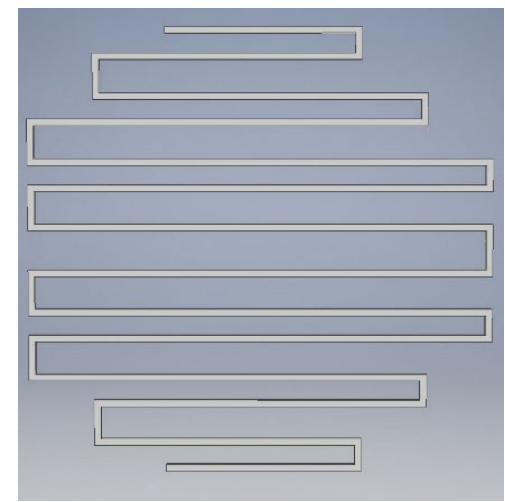

C

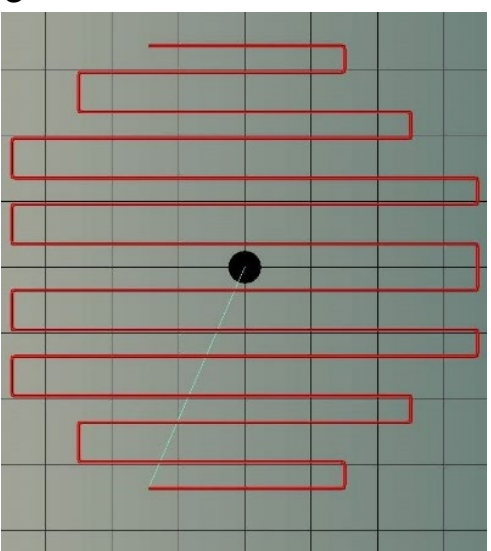

B

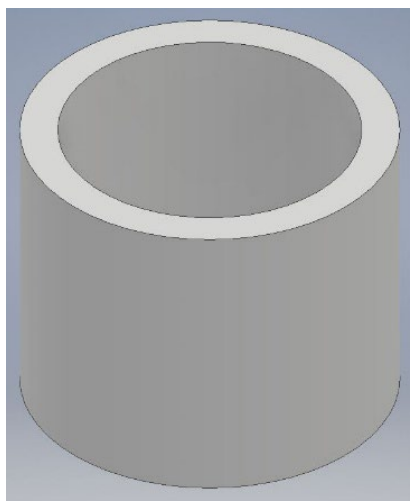

D

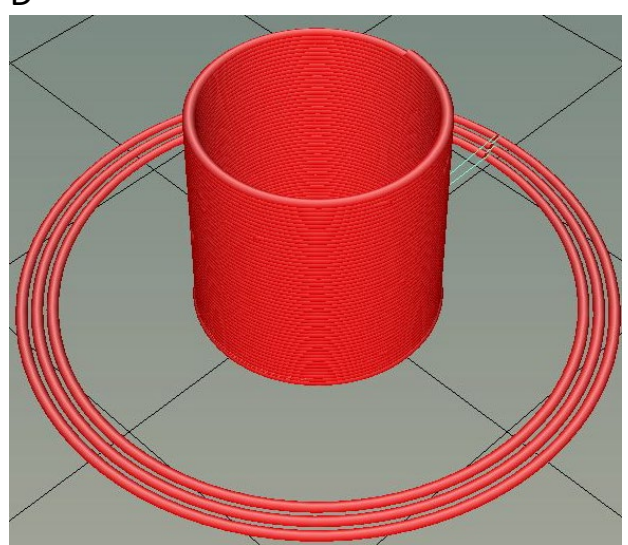

Figure S1. CAD of the printed objects. (A) Computer-aided-design of the printed 2D pattern. (B) Computer-aided-design of the printed 3D pattern. (C) Graphic representation of the GCode used to print the 2D pattern. (D) Graphic representation of the GCode used to print the 3D pattern 


\section{A Carboxylated Agarose - Stiff}
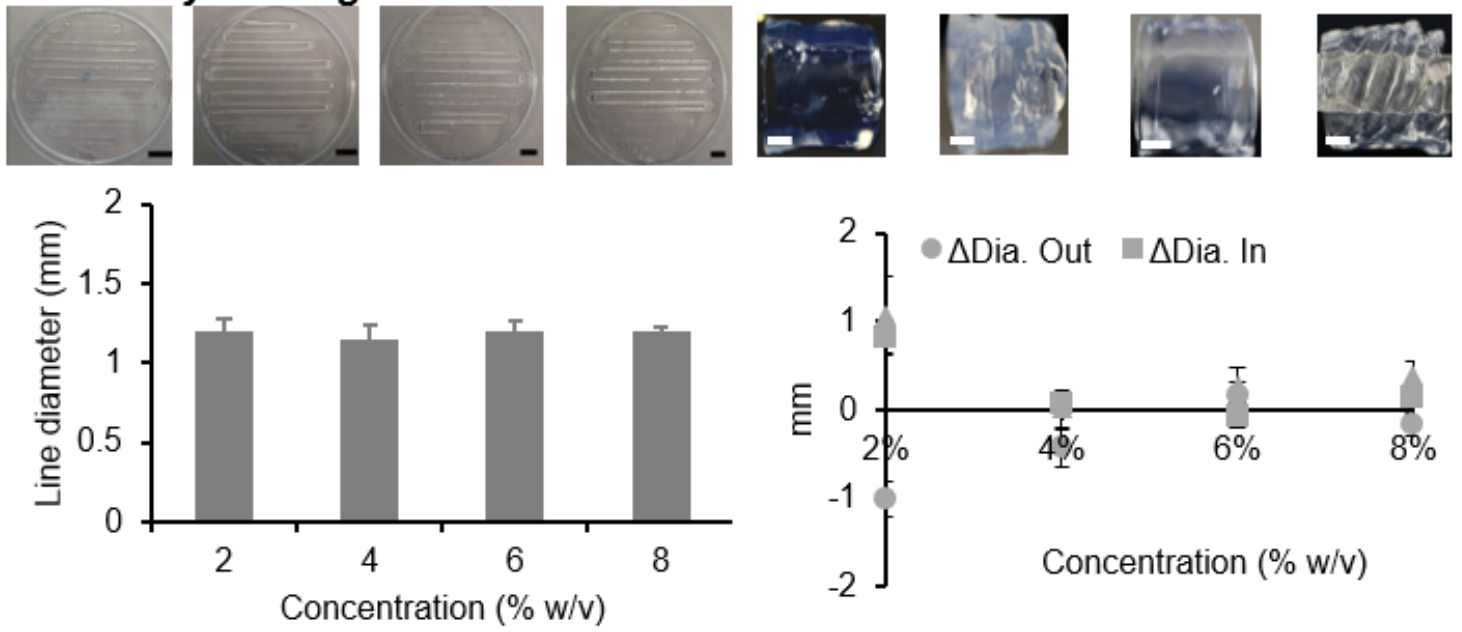

\section{B Carboxylated Agarose - Medium}
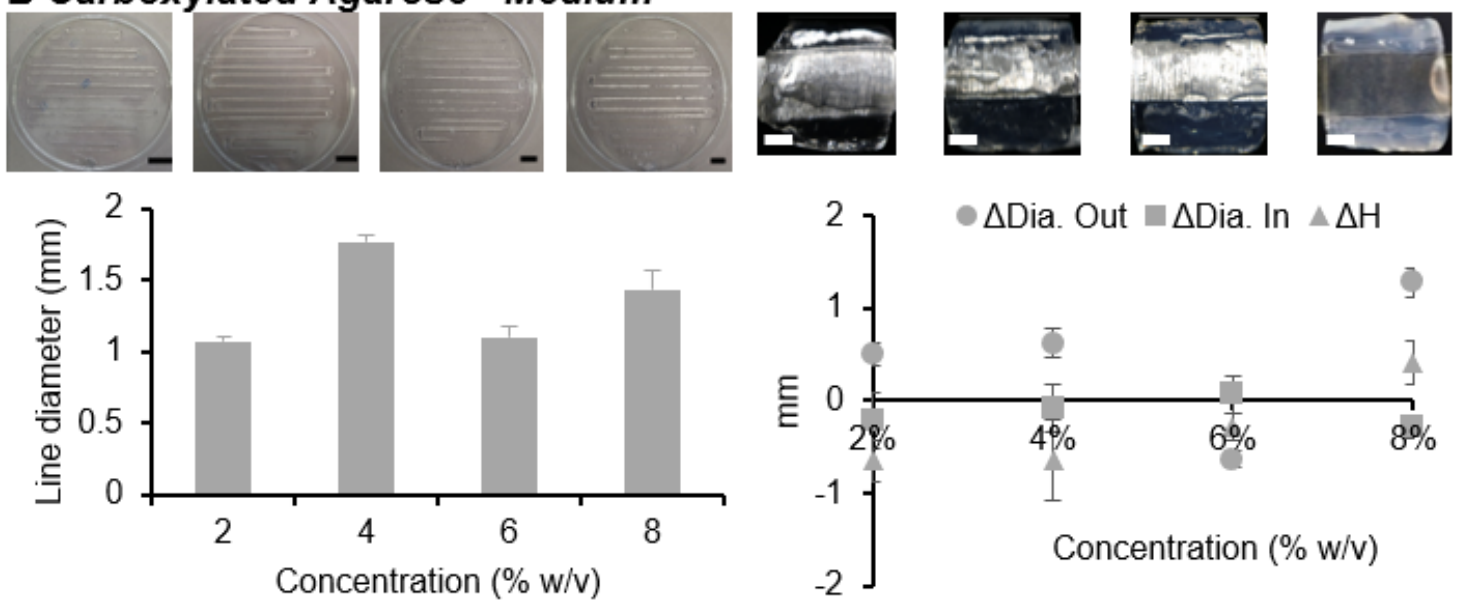

C Carboxylated Agarose - Soft
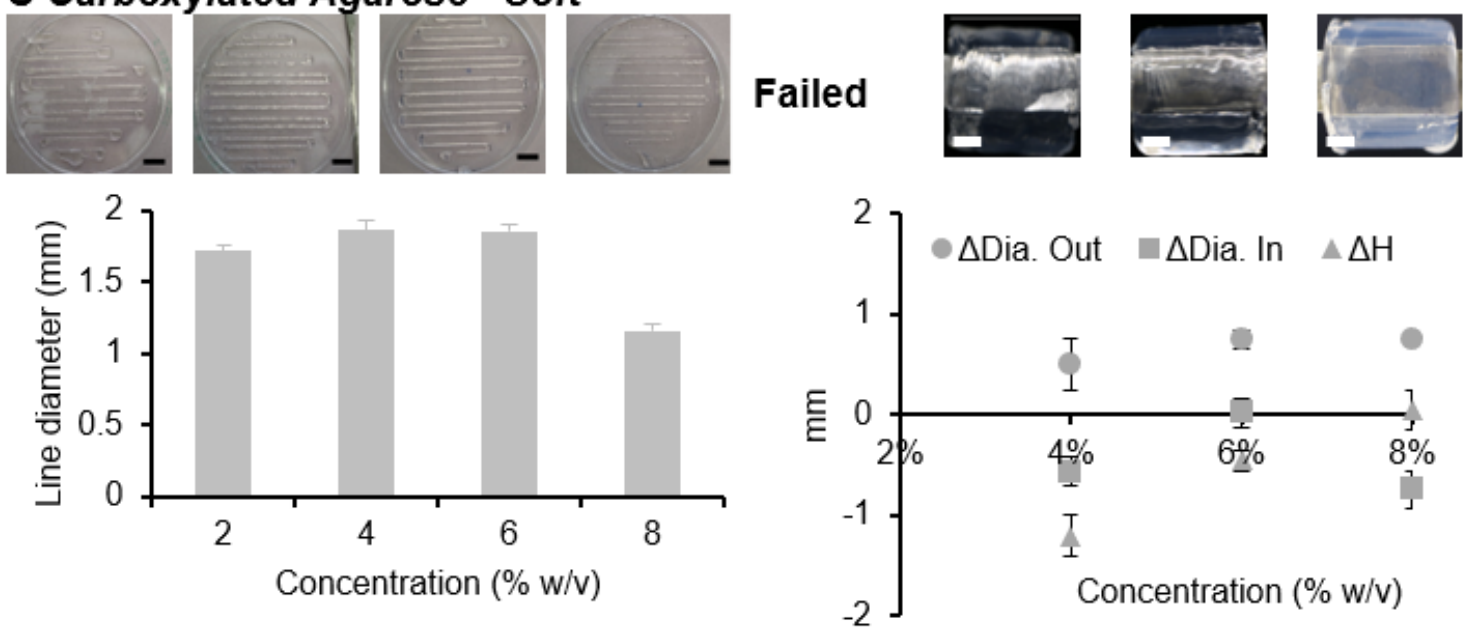

Figure S2. Optimization of printing parameter. The printing parameters were optimised for 2D serpentine lines (left) by measuring the line diameter, and for the 3D cylinders (right) by computing the difference between the dimension of the CAD file and printed object for the cylinders outer $(\Delta d($ out $))$ and inner $(\Delta d($ in) $)$ diameter and height $(\Delta h)$, for different concentration of (A) stiff, (B) medium and $(C)$ soft carboxylated agarose. Error bars represent standard deviation for $n=9$. Scale bar: $10 \mathrm{~mm}$ (left panels) and $2 \mathrm{~mm}$ (right panels) 


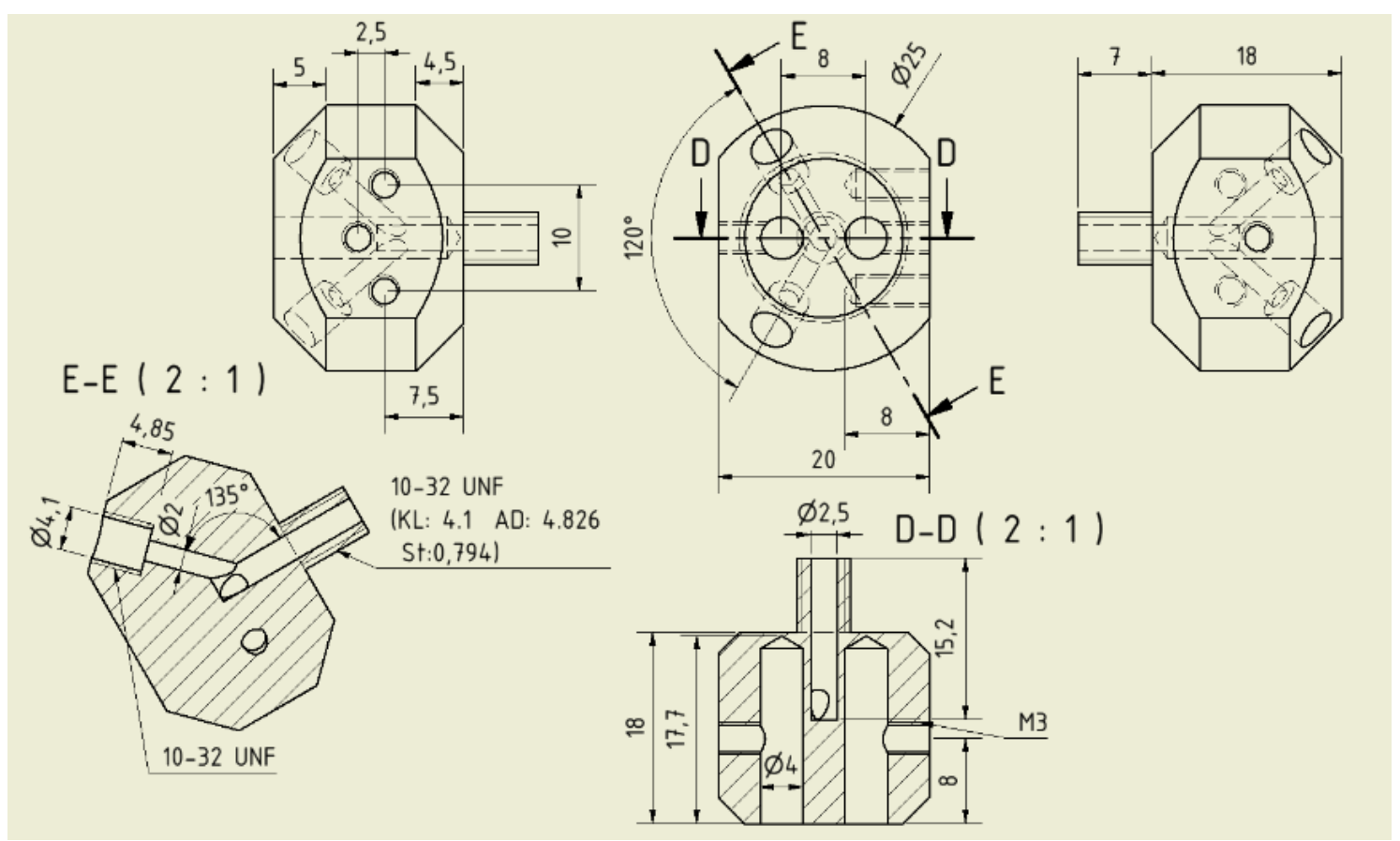

Figure S3. Technical drawing of the mixing head.

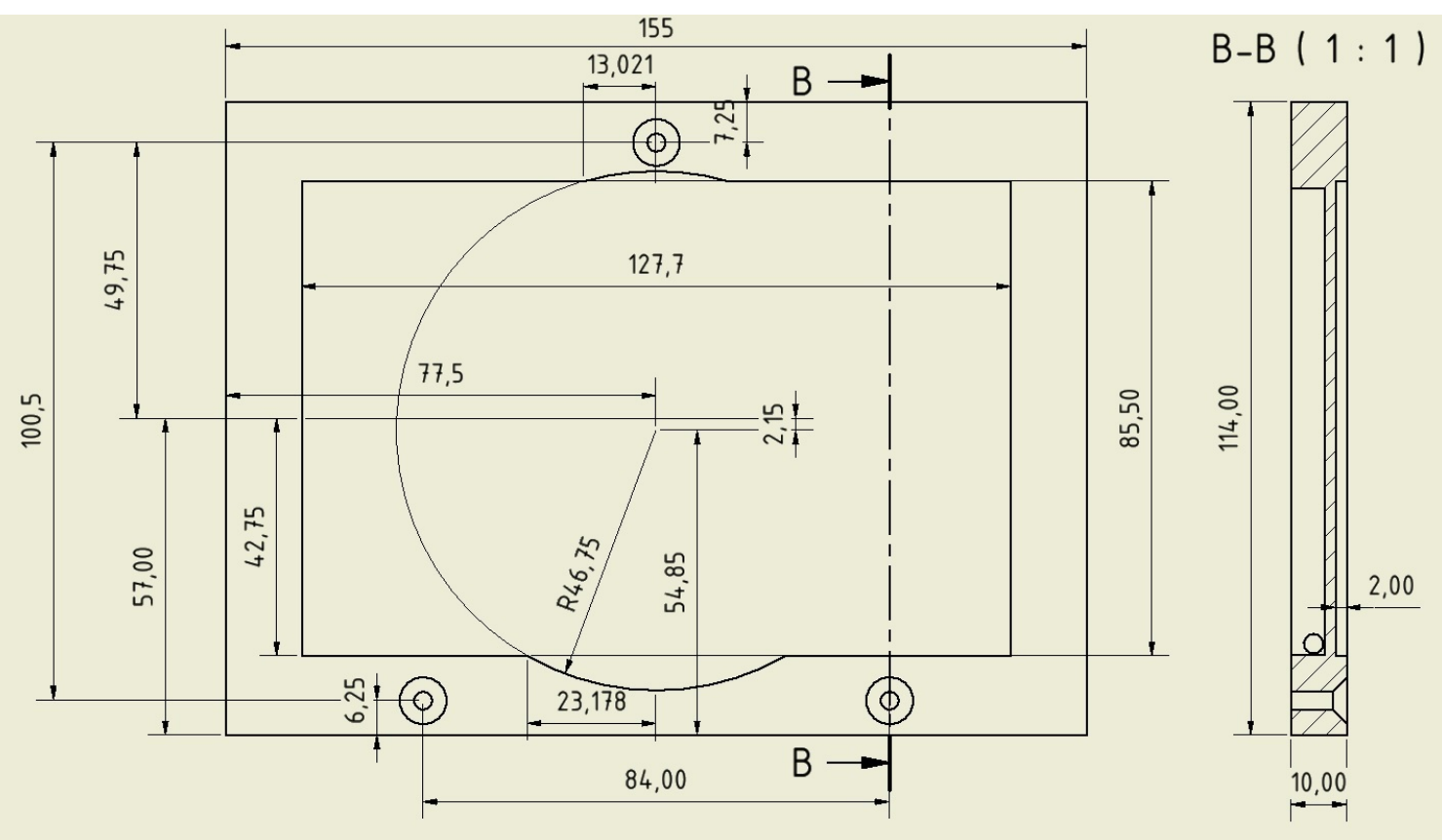

Figure S4. Technical drawing of the cooling stage. 


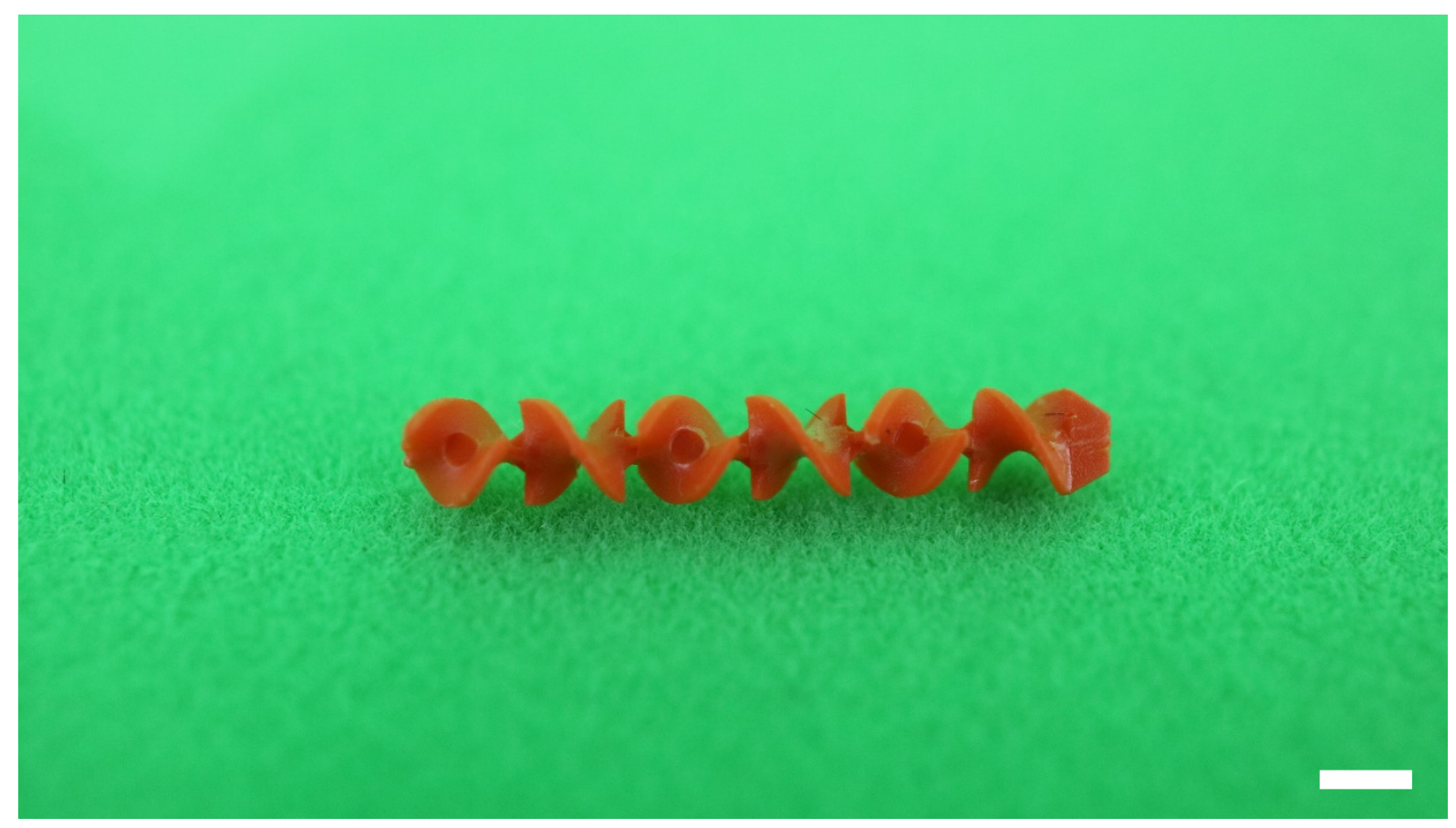

Figure S5. Static mixer. Picture of the mixing element used in this study. Scale bar $2 \mathrm{~mm}$. 
Table S1. Flow rate parameters for 2D Bioprinting of graded mechanical properties Distance from the starting point $(\mathrm{mm})$ Stiff or Medium CA Soft

\begin{tabular}{lcc}
\hline \hline 0 & 0.2 & 0 \\
75 & 0.1 & 0.1 \\
426 & 0 & 0.2
\end{tabular}

Table S2. Flow rate parameters for 3D Bioprinting of graded mechanical properties

\begin{tabular}{lcc} 
& Stiff CA & Soft \\
\cline { 2 - 3 } Layer & \multicolumn{3}{c}{ Flow Rate $(\mathrm{ml} / \mathrm{min})$} \\
\hline $0-16$ & 0.2 & 0 \\
$17-32$ & 0.1 & 0.1 \\
$33-50$ & 0 & 0.1 \\
& Medium CA & Soft \\
\hline \hline $0-16$ & 0.1 & 0 \\
$17-32$ & 0.05 & 0.05 \\
$33-50$ & 0 & 0.1
\end{tabular}

Table S3. Flow rate parameters for 2D Bioprinting of graded concentration of cells.

\begin{tabular}{|c|c|c|}
\hline \multirow[b]{2}{*}{$\begin{array}{l}\text { Distance from the starting } \\
\text { point }(\mathrm{mm})\end{array}$} & Soft CA & HEK293 / Peptide \\
\hline & \multicolumn{2}{|c|}{ Flow Rate (ml/min) } \\
\hline 0 & 0.2 & 0 \\
\hline 75 & 0.15 & 0.05 \\
\hline 279 & 0.1 & 0.1 \\
\hline 503 & 0.05 & 0.15 \\
\hline
\end{tabular}

Table S4. Flow rate parameters for 3D Bioprinting of graded concentration of cells.

\begin{tabular}{lcc} 
& Soft CA & HEK293 / Peptide \\
\cline { 2 - 3 } Layer & \multicolumn{3}{c}{ Flow Rate $(\mathrm{ml} / \mathrm{min})$} \\
\hline \hline $0-10$ & 0.1 & 0 \\
$11-20$ & 0.09 & 0.01 \\
$21-30$ & 0.08 & 0.02 \\
$31-40$ & 0.07 & 0.03 \\
$41-50$ & 0.06 & 0.04
\end{tabular}


A

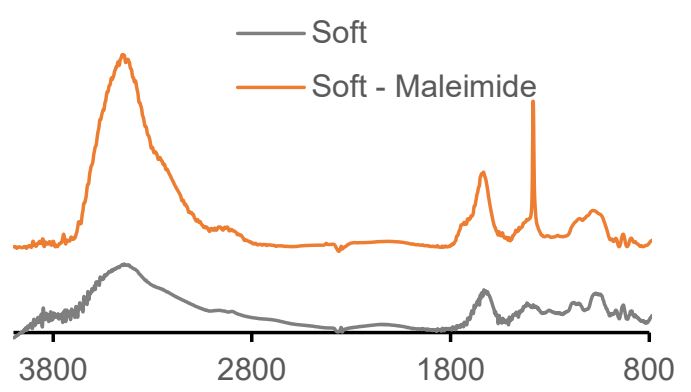

C

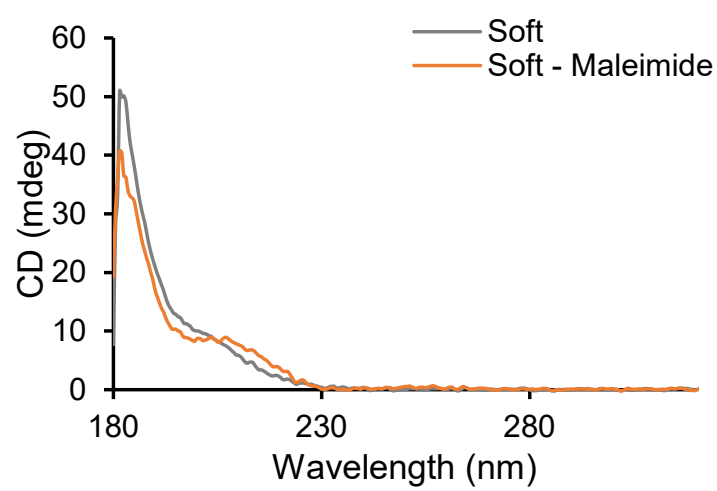

B

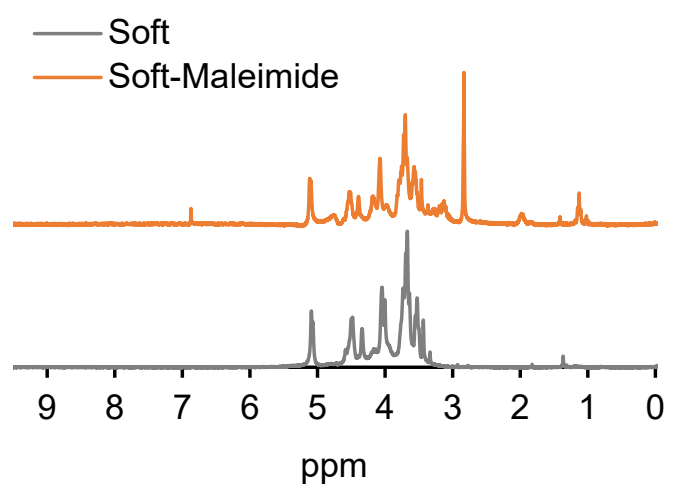

D

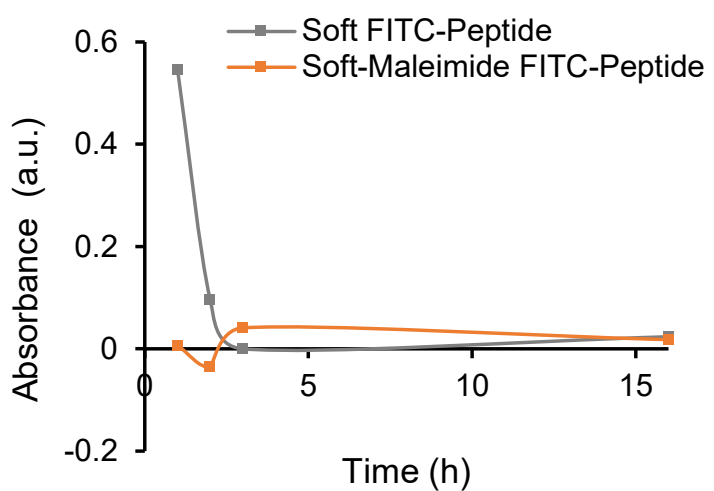

Figure S6. Physico-chemical analysis of the soft carboxylated agarose (CA). CA used in this study compared to the maleimide functionalized soft CA. (A) FTIR, (B) NMR, (C) Circular dichroism. (D) UV absorbance of water incubated with bioprinted object made of CA with FITC-peptide and maleimide functionalized CA with FITC-peptide for different incubation time. 
A Manual mixing

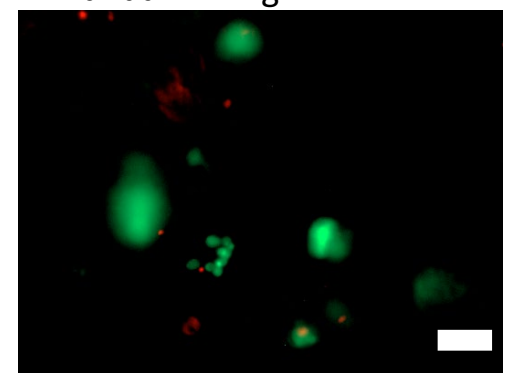

D $0.03 \mathrm{~mL} / \mathrm{min}$

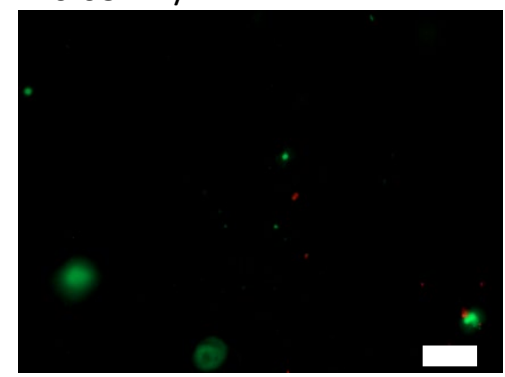

$\mathrm{G} 0.1 \mathrm{~mL} / \mathrm{min}$

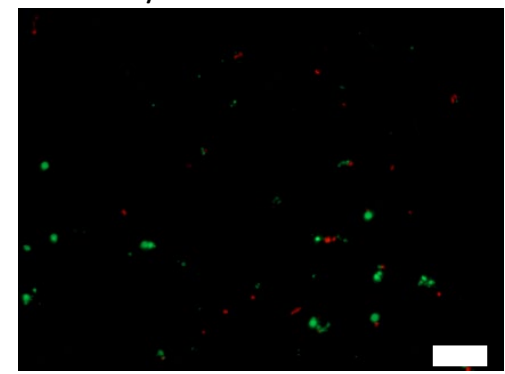

B $0.01 \mathrm{~mL} / \mathrm{min}$

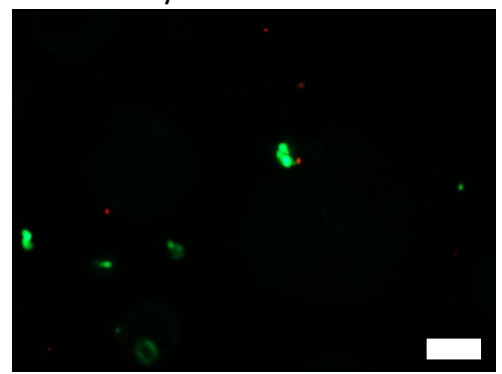

E $0.04 \mathrm{~mL} / \mathrm{min}$

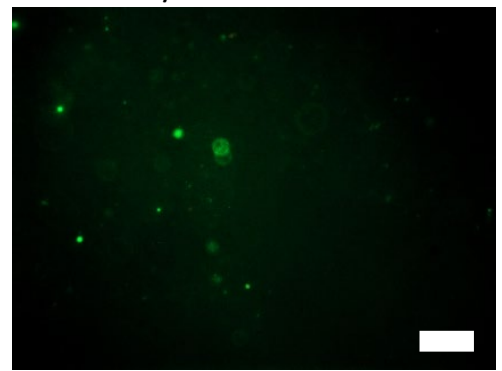

$\mathrm{H} 0.15 \mathrm{~mL} / \mathrm{min}$

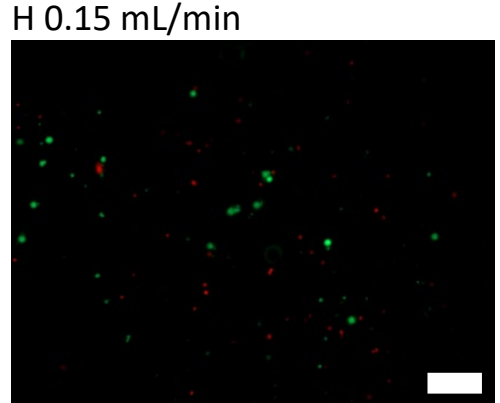

C $0.02 \mathrm{~mL} / \mathrm{min}$

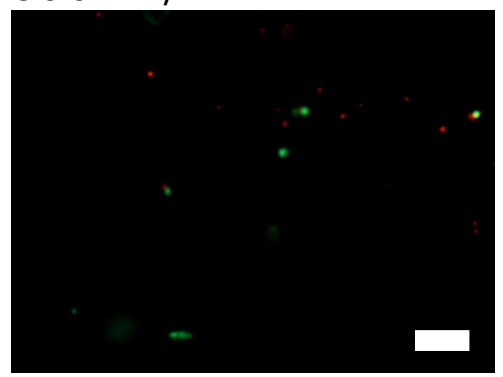

$\mathrm{F} 0.05 \mathrm{~mL} / \mathrm{min}$

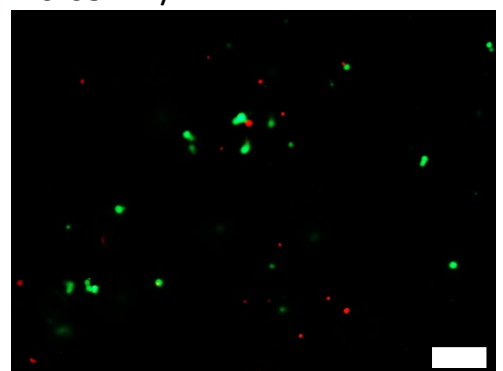

Figure S7. Microscopic picture of LIVE/DEAD assay. Representative images of the LIVE/DEAD assay performed on the CA hydrogel at different extrusion speed. An increase of cell death is observed with an increase of flow rate. While some dead cells are observed when the hydrogel are manually prepared as the CA lacks adhesion motifs. Scale bar $100 \mu \mathrm{m}$. 